\title{
Computational Behavior of Second Law Poiseuille Flow of Micropolar Fluids in a Channel: Analytical Treatment
}

\author{
Priya Mathur $\mathbb{D}^{1},{ }^{1}$ S. R. Mishra $\mathbb{D}^{2},{ }^{2}$ Mahesh Bohra $\mathbb{D D}^{3}$ D. L. Suthar $\mathbb{D}^{4},{ }^{4}$ and S. D. Purohit $\mathbb{D}^{5}$ \\ ${ }^{1}$ Department of Mathematics, Poornima Institute of Engineering \& Technology, Jaipur 302022, India \\ ${ }^{2}$ Department of Mathematics, Siksha O Anusandhan Deemed to be University, Khandagiri 751030, Odisha, India \\ ${ }^{3}$ Department of Mathematics, Govt Mahila Engineering College, Ajmer 05001, India \\ ${ }^{4}$ Department of Mathematics, Wollo University, P.O. Box 1145, Dessie Campus, Dessie, Ethiopia \\ ${ }^{5}$ Department of HEAS (Mathematics), Rajasthan Technical University, Kota 324010, India \\ Correspondence should be addressed to D. L. Suthar; dlsuthar@gmail.com
}

Received 2 May 2021; Revised 24 June 2021; Accepted 24 July 2021; Published 3 August 2021

Academic Editor: Riaz Ahmad

Copyright (C) 2021 Priya Mathur et al. This is an open access article distributed under the Creative Commons Attribution License, which permits unrestricted use, distribution, and reproduction in any medium, provided the original work is properly cited.

\begin{abstract}
The present analysis explores an analytical treatment for the computation of Poiseuille flow of a micropolar fluid in a channel placed in between two horizontal parallel plates. Both the plates are placed at constant wall temperatures. Therefore, the flow region is portioned into two different zones named zone I and zone II. Eringen's micropolar fluid flow phenomena are taking place assuming no-slip conditions at the interface. Suitable nondimensional variables are imposed for the transformation of governing equations. Analytical treatment is carried out employing the in-house symbolic command using the MAPLE software. The behavior of several contributing parameters such as material parameters, the couple stresses for both the zones on the velocity, and microrotation profiles are investigated and presented via graphs. The volume flow rate is also calculated and presented via the tabular form. The major outcomes of the results are presented as the higher the Reynolds number, the rate increases significantly. The profile is tiled near the central region with a pick starting from the lower plate region to the central region in zone I and retards from the central region to the upper plate in the zone II, and the profiles of angular momentum seem to be symmetric in nature about the central region that is shown in both the zones.
\end{abstract}

\section{Introduction}

The knowledge of micropolar fluid has great applicability in many industrial areas and the field of biological research. Recent studies cross over these phenomena on the micropolar fluid by several researchers. The augmentation of both the influences of rotation and inertial between the microelements has been initiated by Eringen [1]. Furthermore, Eringen [2-4] used parallel plates through which the polar fluid past to develop the second law analysis. Eringen proposed a theory for polar fluids which exhibit the rotation of particles in conjunction with the vorticity vector independently and also the action of couple stress. However, such suspended particles into a viscous medium are made of hard particles. In particular, the blood flows in the human body. Ferro fluids are the best examples of micropolar fluids.
Moreover, as described earlier in the field of biological research studies, i.e., the blood flows, colloidal elucidation and their movement, and solutions using the suspension, their application is vital. More precisely, the suspended dumbbellshaped stiff cylindrical components are found in the micropolar fluid, and the governing equations for the fluid flow are based upon the conservation laws of mass, momentum, and constitutive relations. The investigation for the features of several components that characterize the flow using a semiinfinite plate is obtained by Ahmadi [5]. However, they have employed numerical solutions to get the results from the nonlinear ODEs. Recent development carries out the interest for the significant application for the enhancement of heat transfer properties in the polar fluid. The study of certain polymer solutions and the suspension of colloidal particles is vital for chemical engineering 
researchers. Moreover, in the biomedical sciences, complex biological structures are obtained using micropolar fluids. In comparison to conventional fluids, the properties of heat transfer are enhanced by using nanofluid, a new class of heat transfer fluids. The nanoparticles of size $1-100 \mathrm{~nm}$ are suspended into the base fluid to form such nanofluids. Due to the higher thermal conductivity of metallic nanoparticles, the conductivity of the nanofluid is also enhanced. The influence of various thermophysical parameters in the permeable medium for the mixed convection in a nanofluid has been conducted by Ellahi et al. [6]. The characteristics of Brownian motion for the heat transfer properties in a nanofluid are governed by Dogonchi and Ganji [7]. In a conclusive remark, they got that the fluid temperature rises due to an increase in the heat source, and the impact is reversed for thermal radiation. Jena and Mathur [8] worked on similarity solutions for the laminar-free convection flow of the micropolar fluid. They used the shooting method to establish the relationship between various parameters. Murthy and Srinivas [9] discussed entropy generation in steady Poiseuille flow of two immiscible micropolar fluids between two horizontal parallel plates of a channel with constant wall temperatures. The behaviors of several parameters, i.e., micropolarity, couple stress on the velocity, microrotation, and temperature, are discussed. In recent times, many researchers gave attention to micropolar fields [10-14].

Micropolar fluid flow and heat transfer over a nonlinearly stretching plate with viscous dissipation was discussed by Ahmad and Ishak [15]. It is noteworthy that for polymer processing, the role of viscous dissipation is important. The fact is, it behaves like an energy source for the preparation of heat which in turn delays the process of solidification, which is treated as a coolant for the final product. The phenomena of heat and mass transfer are based on the involvement of thermal radiation and chemical reaction. Authors [16] evaluated in their study the interaction of Newtonian heat and mass processes in Walters-B fluid bounded by a moving surface. In past investigations, only the Newtonian heating of heat transfer has been used to examine the features of different fluid models under various aspects and flow geometries. Mathur and Mishra [17] calculated the heat and mass transfer of MHD-free convection through two infinite plates embedded with porous materials. Authors have studied the thermodiffusion effect which is not considered in the previous research. A numerical study for the two-dimensional steady incompressible mixed convective flow of an electrically conductive micro-nanofluid in a stretchable channel was reported by Rauf et al. [18]. They used the Runge-Kutta-Fehlberg fourth-fifth order (RKF45) method to solve the algebraic system of equations with boundary conditions. Mathur and Mishra [19] discussed the problem of MHD boundary layer flow in the presence of radiation and magnetic field over an exponentially stretching sheet. A semianalytical approach was applied by the authors [20] to study the Williamson nanofluid flow through a porous medium in the presence of melting heat transfer boundary conditions [21]. Sundar et al. have given a review on hybrid nanofluids preparation, thermal properties, heat transfer, and friction. To understand the behaviors of melts and many polymer solutions, the Carreau-Yasuda model was established successfully. Hussain et al. [22] performed a numerical study on the Carreau-Yasuda nanofluid model over a convective heated surface near a stagnation point. Many researchers [23, 24] work on entropy generation of nanofluids in a different scenario. The numerical and graphical justification was given to strengthen their work. Bioconvection was imposed to study the rheology of MHD bioconvective nanofluid containing motile microorganisms by Muhammad Awais et al. [25]. Homogeneous and heterogeneous reactions of the $3 \mathrm{D}$ flow of $\mathrm{Cu}$-water and $\mathrm{AL}_{2} \mathrm{O}_{3}$-water nanofluid and entropy generation estimation along stretching cylinder were investigated by Siddiqui and his fellow researchers [26, 27].

The present model is developed for the study on the plane Poiseuille flow of micropolar fluid within a channel formed to be the two horizontal parallel plates. Analytical treatment is carried out for the flow phenomena to get the result using the symbolic routine code by MAPLE. The behavior of no-slip and hyperstick conditions plays a vital role in the velocity and the angular momentum profiles. The computation for the several pertinent parameters is obtained and presented. The numerical results of the volume flow rate, shear stress, and couple stress coefficients are presented via the tabular form.

\section{Problem Formalism}

The plane Poiseuille flow of a micropolar fluid between the two horizontal parallel plates within a channel is considered. Both the plates are extended along the $x$-direction with a fixed distance between them as $2 \mathrm{~h}$. Here, the $x$-axis is treated as the axial, and the $y$-axis is represented as transverse direction with a center of the channel mentioned as the origin (Figure 1). The lower half of the channel is equipped with the region $-h<y<0$ called zone I and the upper half region is $0<y<h$ is known as zone II. It is assumed that the density of the fluid in zone I is heavier than the fluid present in zone II. The governing equations for the incompressible micropolar fluid in both the zones are described following Eringen [2, 4].

Conservation of mass is given as

$$
\frac{\partial \rho}{\partial t}+\nabla \cdot(\rho \vec{q})=0
$$

Conservation of momentum is given as

$$
\begin{aligned}
\rho \frac{\partial \bar{q}}{\partial t}= & \rho \bar{f}-\nabla P-\kappa \nabla \times \bar{v} \\
& -(\mu+k) \nabla \times \nabla \times \bar{q}+(\lambda+2 \mu+\kappa) \nabla(\nabla \cdot \bar{q}) .
\end{aligned}
$$

Conservation of angular momentum is given as $\rho j \frac{\partial \bar{v}}{\partial t}=\rho \bar{l}-\nabla P+\kappa \nabla \times q+\gamma \nabla \times \nabla \times \bar{v}+(\alpha+\beta+\gamma) \nabla(\nabla \cdot \bar{v})$.

Here, the fluid density $\rho$ and the gyration coefficient $j$ are treated as constants, and $P$ is the fluid pressure at any point. 


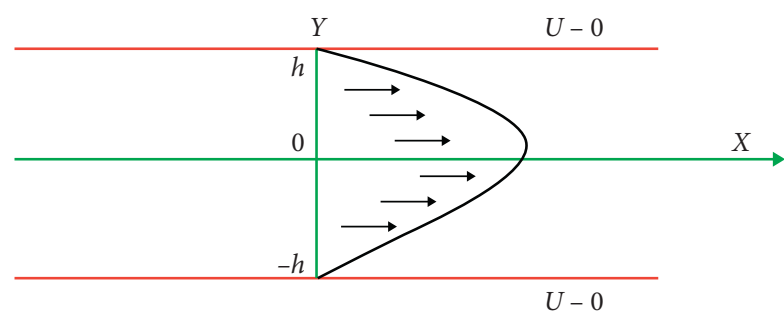

Figure 1: Geometrical configuration.

Also, $\lambda, \mu$, and $\kappa$ are the material constants known as viscosity coefficients, and $\alpha, \beta, \gamma$ are the gyroviscosity coefficients. From equation (3), it is very clear for vanishing $\kappa, \alpha, \beta, \gamma$ and along with $l$ and $f$, the microrotation vanishes. Furthermore, for vanishing $\kappa$, equation (2) for the velocity profiles becomes Newtonian. The stress tensor and the couple stress tensor are given by

$$
\begin{aligned}
t_{i j} & =(-P+\lambda \nabla \cdot q) \delta_{i j}+(2 \mu+\kappa) d_{i j}+\kappa \epsilon_{i j m}\left(\omega_{m}-v_{m}\right), \\
m_{i j} & =\alpha \mathrm{v}_{r, r} \delta_{i j}+\beta v_{i, j}+\gamma v_{i, j},
\end{aligned}
$$

where $v_{i}$ is the microrotating vector, $\omega_{i}$ is the vorticity vector, $d_{i j}$ is the rate of shear strain component, and $\delta_{i j}$ is the Kronecker symbol.

In particular, the Levi-Civita symbol $\epsilon_{i j m}$ is defined as

$$
\epsilon_{i j m}= \begin{cases}-1, & \text { if } i, j, m \text { are cyclic, } \\ 0, & \text { if any two of } i, j, m \text { are equal, } \\ 1, & \text { if } i, j, m \text { are acyclic. }\end{cases}
$$

Also, comma denotes covariant differentiation.

The present model is designed assuming steady, onedimensional incompressible micropolar fluid with a negligible gravity effect.

The velocity and microrotation vector field components are assumed to be $q=(U(Y, 0), 0,0)$ and $\bar{v}=(0,0, C(Y))$, respectively.

The following nondimensional quantities are introduced to transform the governing equations into a dimensionless form:

$$
\begin{aligned}
& x=\frac{X}{h}, \\
& y=\frac{X}{h}, \\
& u==\frac{U}{U_{0}}, \\
& p=\frac{P}{\rho_{1} U_{0}^{2}}, \\
& C=\frac{C U_{0}}{h} .
\end{aligned}
$$

where within the channel, the maximum fluid velocity is $U_{0}$. Employing the aforesaid nondimensional quantities, equation (1) satisfies automatically, and equations (2) and (3) are expressed for the different zones in the following form.

\section{Governing Equations}

The velocity profiles are

$$
u(y)= \begin{cases}u_{1}(y), & -1 \leq y \leq 0(\text { zone }-\mathrm{I}), \\ u_{2}(y), & 0 \leq y \leq 1(\text { zone }-\mathrm{II}),\end{cases}
$$

and the microrotation profile is

$$
\omega(y)= \begin{cases}\omega_{1}(y), & -1 \leq y \leq 0(\text { zone }-\mathrm{I}), \\ \omega_{2}(y), & 0 \leq y \leq 1(\text { zone }-\mathrm{II}) .\end{cases}
$$

3.1. Zone I. In zone I, the transformed equations are

$$
\begin{aligned}
& \frac{\mathrm{d}^{2} u_{1}}{\mathrm{~d} y^{2}}+\left(\frac{\delta_{1}}{1+\delta_{1}}\right) \frac{\mathrm{d} \omega_{1}}{\mathrm{~d} y}=\left(\frac{1}{1+\delta_{1}}\right) \operatorname{Re} \frac{\mathrm{d} p}{\mathrm{~d} x}, \\
& \frac{\mathrm{d}^{2} \omega_{1}}{\mathrm{~d} y^{2}}-s_{1} \frac{\mathrm{d} u_{1}}{\mathrm{~d} y}-2 s_{1} \omega_{1}=0 .
\end{aligned}
$$

3.2. Zone II. Similarly, in zone II, the transformed equations are

$$
\begin{aligned}
& \frac{\mathrm{d}^{2} u_{2}}{\mathrm{~d} y^{2}}+\left(\frac{\delta_{2}}{1+\delta_{2}}\right) \frac{\mathrm{d} \omega_{2}}{\mathrm{~d} y}=\left(\frac{1}{1+\delta_{2}}\right) \frac{n_{p}}{n_{\mu}}-R_{e} \frac{\mathrm{d} p}{\mathrm{~d} x} \\
& \frac{\mathrm{d}^{2} \omega_{2}}{\mathrm{~d} y^{2}}-s_{2} \frac{\mathrm{d} u_{2}}{\mathrm{~d} y}-2 s_{2} \omega_{2}=0,
\end{aligned}
$$

where $\operatorname{Re}=\rho_{1} U_{0} h / \mu_{1}, \delta_{i}=\kappa_{i} / \mu_{i}, s_{i}=\kappa_{i} h^{2} / \gamma_{1}, i=1,2$ and $n_{\mu}=$ $\mu_{2} / \mu_{1}$.

It is clear to understand from the transformed equations that the velocity and microrotation profiles in both the zones are coupled in nature. Since we have considered the
Poiseuille flow, both the plates are fixed and a constant pressure gradient is an act through which the flow is maintained. Here, $\mathrm{d} p / \mathrm{d} x=B$ is a constant. Due to no-slip and hyperstick conditions, the boundary conditions are assumed as 


$$
\begin{aligned}
& u_{1}=0, \quad \omega_{1}=0, \\
& \text { at } y=-1 \\
& u_{1}=u_{2} \text { and } \omega_{1}=\omega_{2} \text {, } \\
& \begin{array}{l}
\left\lceil\frac{\partial u_{1}}{\partial y}+2\left(\frac{\delta_{1}}{1+\delta_{1}}\right)\right. \\
\left.\frac{\mathrm{d} \omega_{1}}{\mathrm{~d} y}\right|_{y=0}=\left.\frac{\mathrm{d} \omega_{2}}{\mathrm{~d} y}\right|_{y=0},
\end{array} \\
& u_{2}=0, \quad \omega_{2}=0, \\
& \frac{\mathrm{d}^{4} u_{1}}{\mathrm{~d} y^{4}}+\left(\frac{2+\delta_{1}}{1+\delta_{1}}\right) s_{1} \frac{\mathrm{d}^{2} u_{1}}{\mathrm{~d} y^{2}}=-\left(\frac{2 s_{1}}{1+\delta_{1}}\right) \operatorname{Re} B, \\
& \omega_{1}=-\frac{1}{2} \frac{\mathrm{d} u_{1}}{\mathrm{~d} y}-\frac{1+\delta_{1}}{2 s_{1} \delta_{1}} \frac{\mathrm{d}^{3} u_{1}}{\mathrm{~d} y^{3}} .
\end{aligned}
$$

3.3. Engineering Coefficients. The shear stress coefficients at the fluid interfaces are expressed as

$$
\tau_{x y}=\left[\frac{\partial u_{i}}{\partial y}+2\left(\frac{\delta_{i}}{1+\delta_{i}}\right) \omega_{i}\right]_{y=o}, \quad i=1,2 .
$$

However, the shear stresses near the lower and upper plates are

$$
\begin{gathered}
\tau_{x y}=\left[\frac{\partial u_{1}}{\partial y}\right]_{y=-1}, \\
\tau_{x y}=\left[\frac{\partial u_{2}}{\partial y}\right]_{y=1} .
\end{gathered}
$$

Similarly, the couple stress coefficients are expressed as

$$
\left.m_{x y}\right|_{\text {zone-I }}=\left.\frac{\mathrm{d} \omega_{1}}{\mathrm{~d} y}\right|_{y=0}, \quad \text { and }\left.m_{x y}\right|_{\text {zone-II }}=\left.n_{\beta} \frac{\mathrm{d} \omega_{2}}{\mathrm{~d} y}\right|_{y=0} \text {. }
$$

3.4. Volumetric Flow Rate. The volumetric flow rate is computed as

$$
q=q_{1}+q_{2}=\int_{-1}^{0} u_{1}(y) \mathrm{d} y+\int_{0}^{1} u_{2}(y) \mathrm{d} y .
$$

\section{Solution of the Problem}

Eliminating $\omega_{1}$ from equations (9) and (10) of zone I, the transformed equations are of the form
Similarly, eliminating $\omega_{2}$ from equations (11) and (12) of zone II, we get

$$
\begin{aligned}
& \frac{\mathrm{d}^{4} u_{2}}{\mathrm{~d} y^{4}}+\left(\frac{2+\delta_{2}}{1+\delta_{2}}\right) s_{2} \frac{\mathrm{d}^{2} u_{2}}{\mathrm{~d} y^{2}}=-\left(\frac{2 s_{2}}{1+\delta_{2}}\right) \frac{n_{\rho}}{n_{\mu}} \operatorname{Re} B, \\
& \omega_{2}=-\frac{1}{2} \frac{\mathrm{d} u_{2}}{\mathrm{~d} y}-\frac{1+\delta_{2}}{2 s_{2} \delta_{2}} \frac{\mathrm{d}^{3} u_{2}}{\mathrm{~d} y^{3}} .
\end{aligned}
$$

However, solving equations (18) and (20), using a symbolic routine command of MAPLE, we get

$u_{1}=C_{1} B_{1} \exp \left(B_{2} y\right)+C_{2} B_{1} \exp \left(-B_{2} y\right)+C_{3} y+C_{4}+B_{3} y^{2}$,

$$
u_{2}=C_{5} B_{4} \exp \left(B_{5} y\right)+C_{6} B_{4} \exp \left(-B_{5} y\right)+C_{7} y+C_{8}+B_{6} y^{2} .
$$

Using the expression of (22) and (23), the results of (19) and (21) can be obtained as

$$
\begin{aligned}
& \omega_{1}=C_{1} B_{7} \exp \left(B_{2} y\right)+C_{2} B_{8} \exp \left(-B_{2} y\right)-0.5 C_{3}-B_{3} y, \\
& \omega_{2}=C_{5} B_{9} \exp \left(B_{5} y\right)+C_{6} B_{10} \exp \left(-B_{5} y\right)-0.5 C_{7}-B_{6} y .
\end{aligned}
$$


where the eight unknowns $C_{i}^{\prime} s, i=1, \ldots, 8$ are to be determined.

Employing the boundary conditions (13) in equations (22)-(25), we get the following matrix to get the unknowns.

$$
\left(\begin{array}{cccccccc}
B_{1} e^{-B_{2}} & B_{1} e^{B_{2}} & -1 & 1 & 0 & 0 & 0 & 0 \\
B_{7} e^{-B_{2}} & B_{8} e^{B_{2}} & -0.5 & 0 & 0 & 0 & 0 & 0 \\
B_{1} & B_{1} & 0 & 1 & -B_{4} & -B_{4} & 0 & -1 \\
B_{7} & B_{8} & -0.5 & 0 & -B_{9} & -B_{10} & 0.5 & 0 \\
B_{13} & B_{14} & -0.5 B_{11} & 0 & -B_{15} & -B_{16} & 0.5 B_{12} & 0 \\
B_{2} B_{7} & -B_{2} B_{8} & -1 & 0 & -B_{5} B_{9} & B_{5} B_{10} & 0 & 0 \\
0 & 0 & 0 & 0 & B_{4} e^{B_{5}} & B_{4} e^{-B_{5}} & 1 & 1 \\
0 & 0 & 0 & 0 & B_{9} e^{B_{5}} & B_{10} e^{-B_{5}} & -0.5 & 0
\end{array}\right)\left(\begin{array}{c}
C_{1} \\
C_{2} \\
C_{3} \\
C_{4} \\
C_{5} \\
C_{6} \\
C_{7} \\
C_{8}
\end{array}\right)=\left(\begin{array}{c}
-B_{3} \\
-B_{3} \\
0 \\
0 \\
0 \\
-B_{6} \\
-B_{6} \\
-B_{6}
\end{array}\right),
$$

where the known values $B_{i}^{\prime} s, i=1, \ldots, 16$ are presented in the appendix.

\section{Results and Discussion}

A computational study on the flow of second law Poiseuille flow for a micropolar fluid within a channel is carried out in the current problem. The non-Newtonian fluid passed through two horizontal plates that were partitioned into two zones separated by the central region. The pressure gradient is assumed to be the constant. The crux of the investigation is the assumption of no-slip and hyperstick boundary conditions. The transformed governing equations for the velocity and the microrotation profiles in both the zones are coupled in nature, and a symbolic routine is handled by the MAPLE to solve the differential equations. The computed results for different characterizing parameters on the flow profiles are presented via graphs, and the numerical computations for the volume flow rate are obtained and displayed through a table. Throughout the computation, the following values are considered to be fixed, except the variation of the particular parameter displayed in the corresponding graphs. The velocity distributions for several parameters are deployed in Figures 2-7, and the microrotation profiles are presented through Figures 8-13. In all the figures, the dotted line indicates the variation of the parameters in the zone I and the bold line represents the variation in zone II.

5.1. Velocity Profiles. Figure 2 elaborates the behavior of the cross-viscosity parameter $\delta_{1}$ on the velocity profiles. From the governing equations, it is clear to see that for large $\delta_{1}$, the fluid particles rotate about themselves with high angular velocity. Therefore, the velocity profile retards both the regions resulted in the channel thickness increase. It is interesting to observe that the profile picks near the lower plate up to the central region, and thereafter, the fall in the profile is marked from the central region to the upper plate region. The profile behavior validates with the work of Umavati et al. [28]. The trend of the graph in both the regions is a very similar pattern to that of the earlier study. Finally, it is concluded that increasing cross-viscosity or the micropolarity suitably reduces the velocity profiles in both zones. The influence of the couple stress parameter $s_{1}$ on the velocity profiles for fixed values of other pertinent parameters is displayed in Figure 3. The distributions in both zones are displayed significantly. It is clearly visible that increasing couple stress parameter, the profile increases, showing the channel with decreases at both the plates. The large value of couple stress characterizes the Newtonian case. The couple stress tensor occurs due to the rotation of the particles. Figures 4 and 5, respectively, present the variation of crossviscosity $\delta_{2}$ and the couple stress parameter $s_{2}$ on the velocity distributions. As described earlier, for the increasing micropolarity parameter, the velocity profile retards significantly in both the zones, whereas the reverse impact is rendered for the increasing couple stress. However, the increasing couple stresses the increase in the velocity distribution is insignificant. Reynolds number characterizes the relation between the inertial and the viscous force. From the mathematical form of the Reynolds number, it is observed that as inertial force increases, the Reynolds number increases. The role of Reynolds number is an important aspect of the velocity distribution that is presented in Figure 6. The higher the Reynolds number, the rate increases significantly. The profile is tiled near the central region with a pick starting from the lower plate region to the central region in zone I and retards from the central region to the upper plate in zone II. The physical behavior of the Reynolds number shows as a controlling parameter for the flow phenomena. Dual characteristics of the constant pressure gradient are marked on the velocity distribution in both the zones that are reflected in Figure 7. Flow separation occurs due to a change in pressure gradient. Adverse pressure gradient presents, if the pressure is acted along the direction of flow. In zone I, for an increase in negative pressure gradient, the rise in velocity profile is marked, whereas the backflow occurs with increasing pressure gradient. A similar observation is reflected in zone II for various values of a pressure gradient.

5.2. Angular Velocity Profiles. The characteristics of pertinent physical parameters on the angular velocity are 


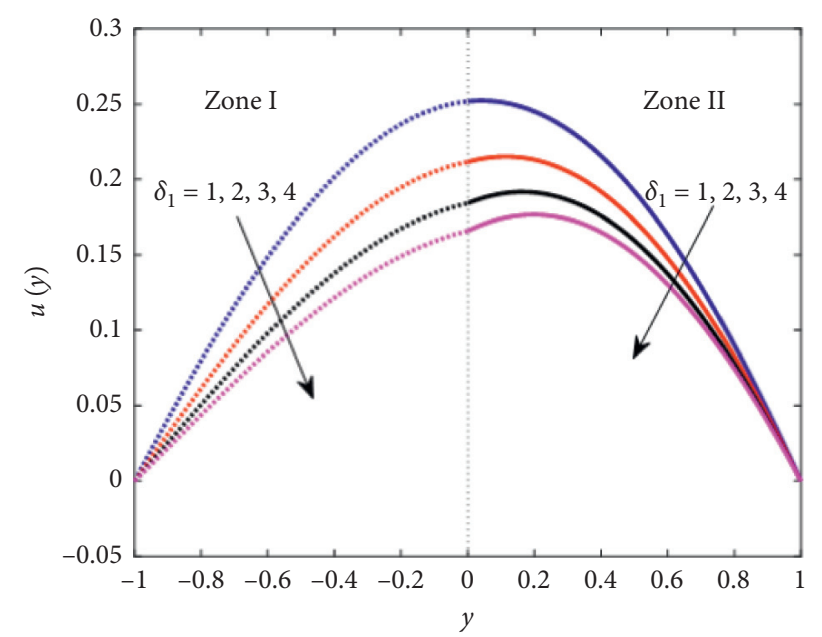

Figure 2: Velocity profiles for various $\delta_{1}$.

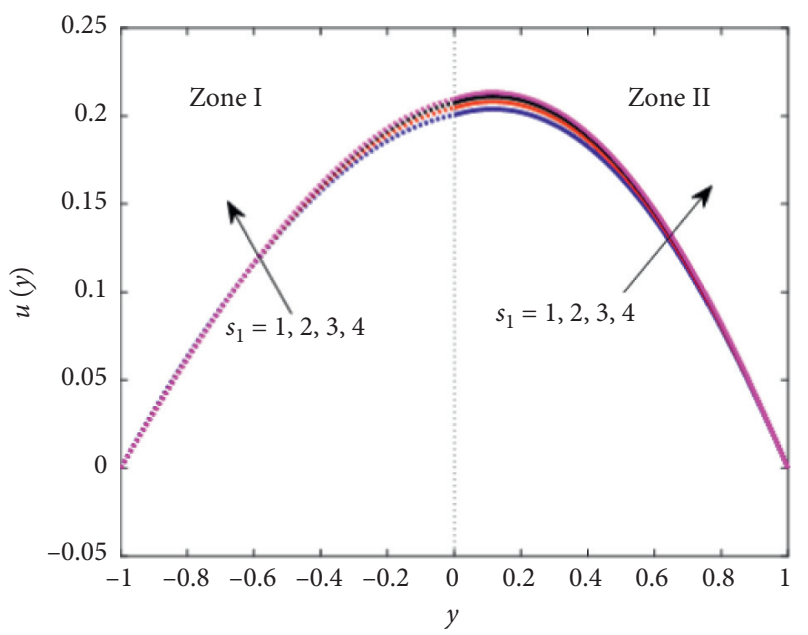

FIgURE 3: Velocity profiles for various $s_{1}$.

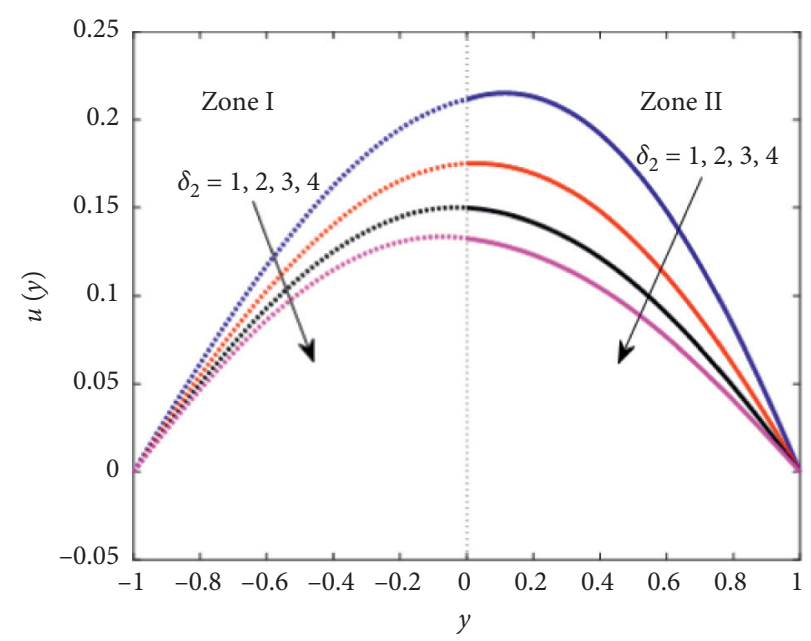

Figure 4: Velocity profiles for various $\delta_{2}$. 


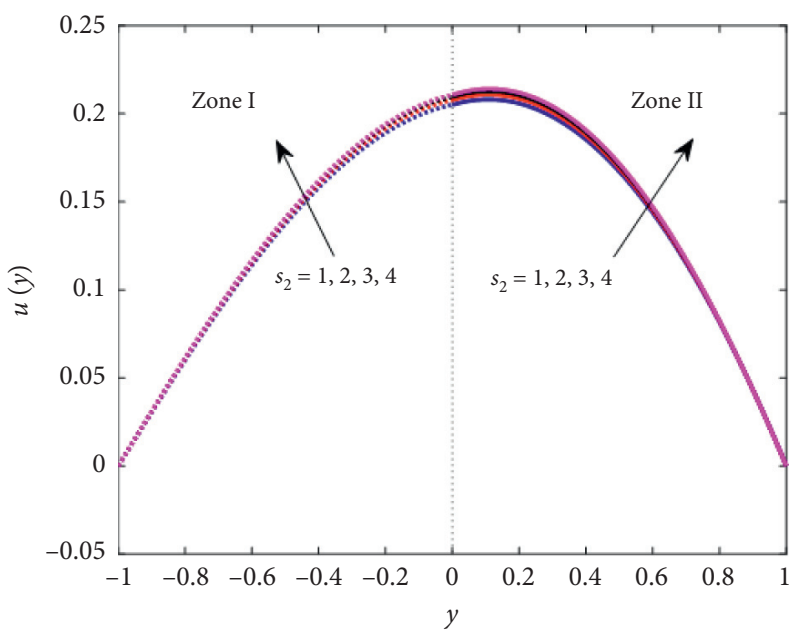

Figure 5: Velocity profiles for various $s_{2}$.

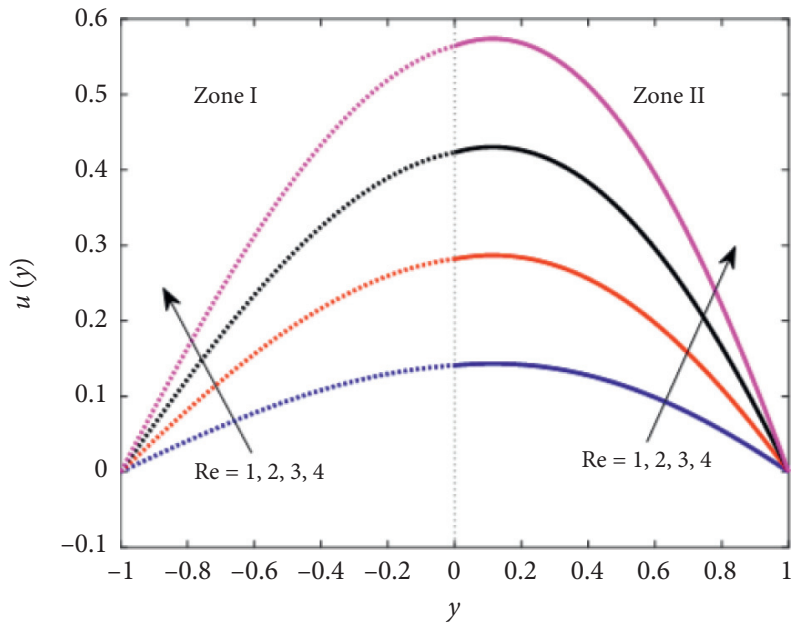

Figure 6: Velocity profiles for various Re.



Figure 7: Velocity profiles for various $\mathrm{d} p / \mathrm{d} x$. 


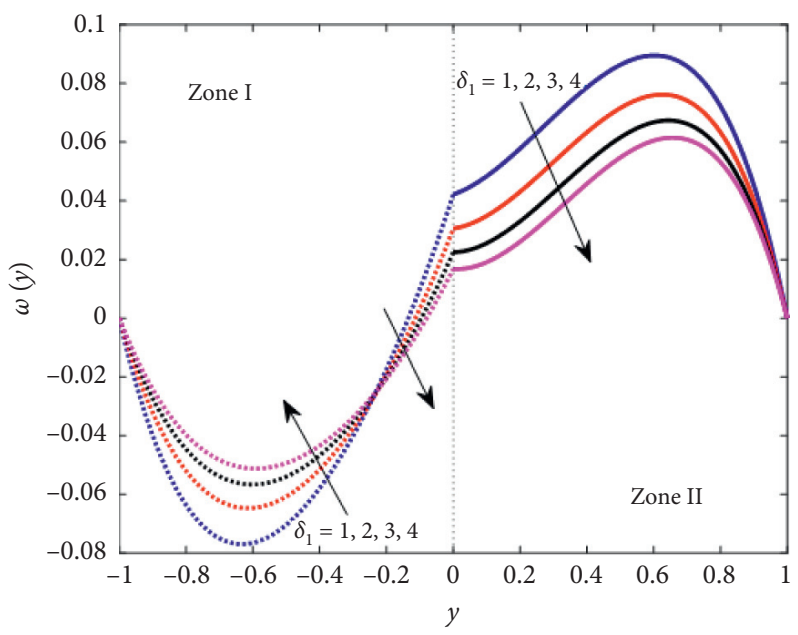

Figure 8: Microrotation profiles for various $\delta_{1}$.

displayed in Figures 8-13. The behavior of cross-viscosity $\delta_{1}$ is displayed in Figure 8. In zone I, the profile behaves in the opposite phenomenon from the point of inflection. The increase in micropolarity increases the profile in the first half, whereas the reverse impact is observed in the second half. However, the backflow occurs in zone I. Due to the rotation of the particles, in zone II, the increase in cross-viscosity retards the angular velocity significantly. Near the central region, the pick in the profile is marked, and with a successive fall, the profile leads towards the upper plate. Figure 9 exhibits the variation of couple stress $s_{1}$ on the angular velocity profiles. Initiation with a backflow, the profile enhances irrespective the values of $s_{1}$ towards the central region, but for the increasing $s_{1}$, the profiles of angular velocity retards. Moreover, in zone II, the profile behaves with a similar trend in the positive domain. The variation of the cross-viscosity $\delta_{2}$ and the couple stress $s_{2}$ on the angular velocity is displayed in Figures 10 and 11, respectively. The profile behaves oppositely in different zones. Exhibiting a backflow in zone I, the angular velocity enhances with increasing $\delta_{2}$, and retardation is marked throughout the positive domain in zone II. For the variation $s_{2}$ in zone I, the change in angular velocity is very negligible. In a close remark, it is seen that with a backflow, the dual behavior is highlighted. However, in zone II, the attenuate in angular velocity is shown with enhancing couple stress parameter. Figure 12 portrays the contribution of the Reynolds number on the profiles for the fixed values of other parameters. As described earlier about the physical significance of the Reynolds number, the increasing value retards the angular momentum drastically in zone I. However, in zone II, the profile enhances in similar magnitude in the positive domain. The characteristics of the pressure gradient are exhibited on the angular velocity that is presented in Figure 13. The profiles of angular momentum seem to be symmetric in nature about the central region that is shown in both the zones. It is clear to see that for the increase in pressure gradient from negative to positive, the profile enhances significantly in zone I and in zone II, and the



Figure 9: Microrotation profiles for various $s_{1}$.

impact is reversed. Therefore, the symmetricity in the profiles is rendered.

5.3. Engineering Coefficients. Finally, the shear stress coefficients, as well as the couple stress coefficients at the fluid interface, are computed for various parameters and are displayed in Table 1. It is seen that the increase in crossviscosity/micropolarity parameters retards the rate of shear stress and couple stress near the fluid interface. Whereas, reverse impact is observed with the increase in couple stress parameters $s_{1}$, i.e., the rate coefficients are enhanced. It is also pointed that the rate of shear stress coefficient increases, but the rate of couple stress coefficient decreases for increasing couple stress parameter $s_{2}$. Also, the Reynolds number parameter enhances both the rate coefficients significantly. Table 2 displays the rate of shear stress coefficients at the lower as well as the upper plate for different contributing parameters. The rate of shear stress at the lower plate attenuates in magnitude with the increasing crossviscosity parameter, whereas the retardation is marked at the 


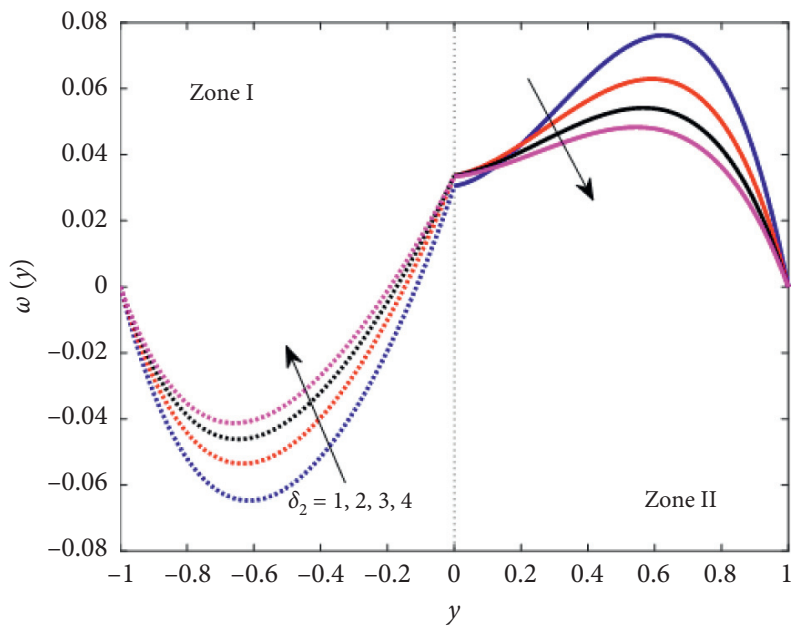

FIgURE 10: Microrotation profiles for various $\delta_{2}$.

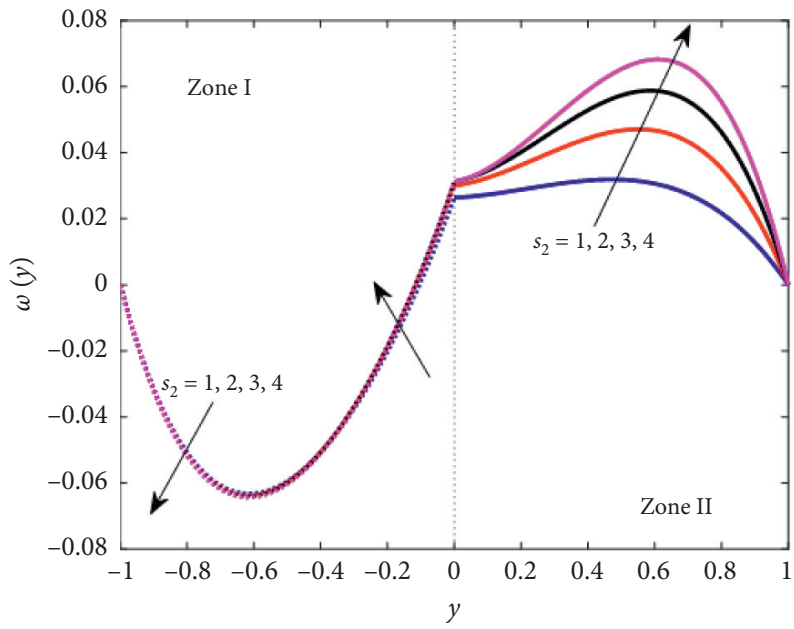

FIgURE 11: Microrotation profiles for various $s_{2}$.

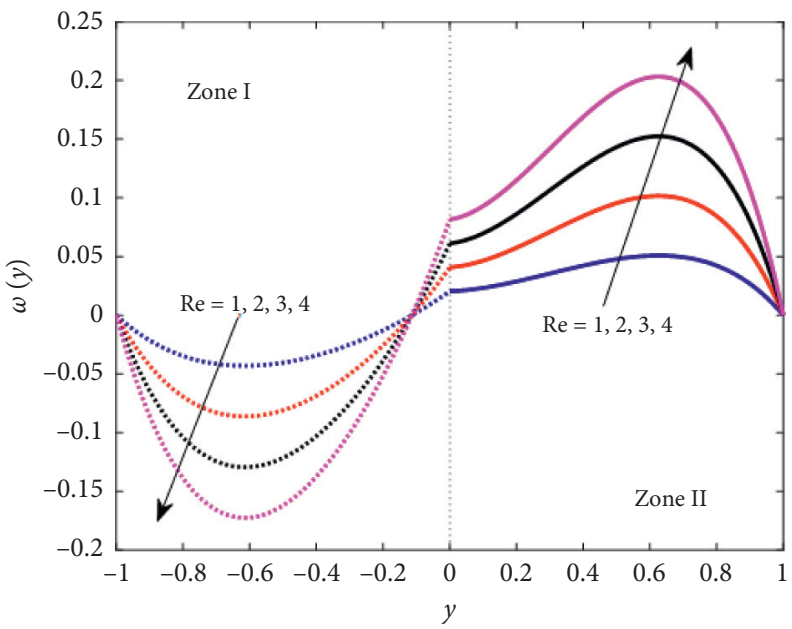

FIgURE 12: Microrotation profiles for various Re. 


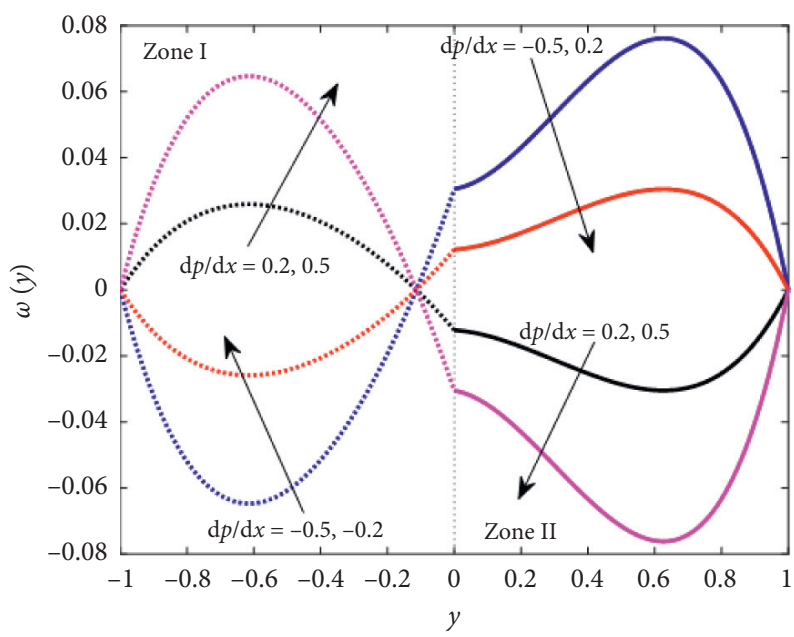

FIGURE 13: Microrotation profiles for various $\mathrm{d} p / \mathrm{d} x$.

TABLE 1: Rate of shear stress coefficients and the couple stress coefficients at the fluid interfaces.

\begin{tabular}{ccccccccc}
\hline$\delta_{1}$ & $s_{1}$ & $\delta_{2}$ & $s_{2}$ & $\operatorname{Re}$ & $\left(\partial u_{1} / \partial y\right)+2\left(\delta_{1} /\left(1+\delta_{1}\right)\right) \omega_{1}$ & $\left(\partial u_{2} / \partial y\right)+2\left(\delta_{2} /\left(1+\delta_{2}\right)\right) \omega_{2}$ & $\partial \omega_{1} / \partial y$ & -0.042152147 \\
\hline 1 & 5 & 1 & 5 & 1.5 & -0.209728562 & -0.209728562 & -0.033721718 \\
2 & & & & & -0.170874742 & -0.161806471 & -0.03053535 & -0.022472561 \\
3 & & & & & -0.150570191 & -0.148996849 & -0.016792852 & -0.013434281 \\
4 & & & & & -0.138921138 & -0.149083602 & -0.089612426 & -0.071689941 \\
1 & 1 & & & & -0.149083602 & -0.173254566 & -0.070460517 & -0.056368414 \\
& 2 & & & & -0.173254566 & -0.189489377 & -0.057741527 & -0.046193222 \\
& 3 & & & & -0.189489377 & -0.201078539 & -0.048768797 & -0.039015037 \\
& 4 & & & & -0.201078539 & -0.209728562 & -0.042152147 & -0.033721718 \\
& 5 & 1 & & & -0.209728562 & -0.142742409 & -0.042615749 & -0.034092599 \\
& 2 & & & -0.156947658 & -0.10486528 & -0.04178063 \\
& 3 & & & -0.125755595 & -0.033424504 \\
& 4 & & & -0.105594402 & -0.210613768 & -0.040960015 & -0.032768012 \\
& 1 & 1 & & -0.210613768 & -0.208181944 & -0.032808813 & -0.02624705 \\
& & 2 & & -0.208181944 & -0.207801688 & -0.038287983 & -0.030630386 \\
& & 3 & & -0.207801688 & -0.208481457 & -0.040910744 & -0.032728595 \\
& & 4 & & -0.208481457 & -0.139819042 & -0.041979635 & -0.033583708 \\
& & 5 & 1 & -0.139819042 & -0.279638083 & -0.028101432 & -0.022481145 \\
& & & 2 & -0.279638083 & -0.419457125 & -0.056202863 & -0.04496229 \\
& & & & -0.419457125 & -0.559276166 & -0.084304295 & -0.067443436 \\
& & & -0.559276166 & & -0.112405726 & -0.089924581 \\
\hline
\end{tabular}

TABLE 2: The rate of shear stress coefficients at the lower and upper plates, respectively.

\begin{tabular}{|c|c|c|c|c|c|c|}
\hline$\delta_{1}$ & $s_{1}$ & $\delta_{2}$ & $s_{2}$ & $\operatorname{Re}$ & $\partial u_{1} / \partial y$ & $\partial u_{2} / \partial y$ \\
\hline 1 & 5 & 1 & 5 & 1.5 & $-2.22 E-16$ & $5.55 E-17$ \\
\hline 2 & & & & & $8.33 E-17$ & 0 \\
\hline \multirow[t]{8}{*}{1} & 1 & & & & $-5.55 E-17$ & $-5.55 E-17$ \\
\hline & 2 & & & & $8.60 E-16$ & $6.66 E-16$ \\
\hline & 5 & 1 & & & $-2.22 E-16$ & $5.55 E-17$ \\
\hline & & 2 & & & $3.61 E-16$ & $-2.78 E-17$ \\
\hline & & 1 & 1 & & $2.22 E-16$ & $3.05 E-16$ \\
\hline & & & 2 & & $0.00 E+00$ & $2.22 E-16$ \\
\hline & & & 5 & 1 & $-1.39 E-16$ & $5.55 E-17$ \\
\hline & & & & 2 & $-2.78 E-16$ & $1.11 E-16$ \\
\hline
\end{tabular}


TABLE 3: The computation of volumetric flow rate.

\begin{tabular}{|c|c|c|c|c|c|}
\hline$\delta_{1}$ & $s_{1}$ & $\delta_{2}$ & $s_{2}$ & $\mathrm{Re}$ & $q=q_{1}+q_{2}$ \\
\hline 1 & 5 & 1 & 5 & 1.5 & 0.335686766 \\
\hline 2 & & & & & 0.249661868 \\
\hline \multirow[t]{8}{*}{1} & 1 & & & & 0.351164688 \\
\hline & 2 & & & & 0.344302096 \\
\hline & 5 & 1 & & & 0.335686766 \\
\hline & & 2 & & & 0.302175536 \\
\hline & & 1 & 1 & & 0.305201271 \\
\hline & & & 2 & & 0.312959388 \\
\hline & & & 5 & 1 & 0.223791177 \\
\hline & & & & 2 & 0.223791177 \\
\hline
\end{tabular}

upper plate. A distinct characteristic is marked in the rate of shear stress for a couple of stress parameters. An increase in $s_{1}$ creases the shear stress at both the plates, whereas increasing $s_{2}$ retardation is marked. With the increasing Reynolds number, the rate increases in magnitude at the lower plate, and the effect is opposite at the upper plate. Moreover, Table 3 escalates the volumetric flow rate for various contributing parameters. The flow rate decreases with the increase in micropolarity parameters as well as the couple stress parameter $s_{1}$, and increasing $s_{2}$, the flow rate increases significantly.

\section{Conclusive Remarks}

The present investigation was carried out for the second law analysis of the plane Poiseuille flow of micropolar fluid within two horizontal plates. Analytical treatment is obtained with the help of symbolic command of MAPLE software, and computation of the flow phenomena characterized by various parameters is displayed and elaborated. However, the conclusive remarks are laid as follows.

An increase in channel thickness is marked for the increase in cross-viscosity parameters

The reverse impact is rendered for an increasing couple of stress parameters

Reynolds number is favorable for the attenuation in the velocity profiles

Dual character is attributed in the profiles of angular momentum for increasing Reynolds number in both zones

Retardation in the rate of shear stress is marked for the increment in the cross-viscosity parameters

The volume flow rate decreases for increasing viscosity parameters

Last but not least, several interesting studies can be developed using the same model to carry out further investigation. Various applications depend upon the second law analysis since no process can transfer heat from a cooler body to a hotter body on its own. The process of refrigeration and cooling engine is based on second law analysis. Though we have considered the constant viscosity properties, therefore, one can assume variable viscosity along with thermal conductivity for their investigation.

\section{Appendix}

$$
\begin{aligned}
& B_{1}=\frac{1+\delta_{1}}{s_{1}\left(2+\delta_{1}\right)}, \\
& B_{2}=\sqrt{\frac{s_{1}\left(2+\delta_{1}\right)}{1+\delta_{1}}}, \\
& B_{3}=\frac{B}{\left(2+\delta_{1}\right)} \text {, } \\
& B_{4}=\frac{1+\delta_{2}}{s_{2}\left(2+\delta_{2}\right)} \\
& B_{5}=\sqrt{\frac{s_{2}\left(2+\delta_{2}\right)}{1+\delta_{2}}}, \\
& B_{6}=\frac{n_{p} B}{n_{g}\left(2+\delta_{2}\right)} \text {, } \\
& B_{7}=-\frac{1}{2} B_{1} B_{2}-\frac{1+\delta_{1}}{2 \delta_{1} s_{1}} B_{1} B_{2}^{3} \text {, } \\
& B_{8}=\frac{1}{2} B_{1} B_{2}+\frac{1+\delta_{1}}{2 \delta_{1} s_{1}} B_{1} B_{2}^{3} \text {, } \\
& B_{9}=-\frac{1}{2} B_{4} B_{5}-\frac{1+\delta_{2}}{2 \delta_{2} s_{2}} B_{4} B_{5}^{3} \text {, } \\
& B_{10}=\frac{1}{2} B_{4} B_{5}+\frac{1+\delta_{2}}{2 \delta_{2} s_{2}} B_{4} B_{5}^{3} \text {, } \\
& B_{11}=\frac{2 \delta_{1}}{\left(1+\delta_{1}\right)}, \\
& B_{12}=\frac{2 \delta_{2}}{1+\delta_{2}}, \\
& B_{13}=B_{1} B_{2}+B_{11} B_{7} \text {. } \\
& B_{14}=-B_{1} B_{2}+B_{11} B_{8} \text {, } \\
& B_{15}=B_{4} B_{5}+B_{12} B_{9} \text {, } \\
& B_{16}=-B_{4} B_{5}+B_{12} B_{10} \text {, }
\end{aligned}
$$




\section{Nomenclature}

$\begin{array}{ll}\text { Be: } & \text { Bejan number } \\ \text { Br: } & \text { Brinkman number } \\ \bar{q}: & \text { Velocity vector } \\ a_{j}: & \text { Microinertia parameter } \\ \text { Bi: } & \text { Biot number }\end{array}$

$\mathrm{Br} / \Omega$ : Viscous dissipation parameter

$D$ : Deformation tensor

Cp: Specific heat

Gr: Grashof number

$h$ : Channel width

$k_{f}$ : Thermal conductivity

$m^{2}$ : Micropolar parameter

$N$ : Coupling number

Nh: Entropy generation (heat transfer)

Ns: Entropy generation number

Nv: Entropy generation (viscous dissipation)

Re: Reynolds number

$T$ : Dimensional temperature

$n_{\beta}$ : Ratio of couple stress viscosity coefficients

$n_{k}$ : Ratio of thermal conductivities

$n_{\mu}$ : Ratio of viscosities

$T_{1}$ : Ambient temperature

$T_{2}$ : $\quad$ Fluid temperature

$u$ : Dimensional axial velocity

$X: \quad$ Flow direction

$Y$ : Normal to the flow direction.

Greek Symbols

$\alpha$ : Inclined angle

$\alpha_{1}$ : Slip parameter

$\beta, \gamma$ : Gyration viscosity coefficients

$\kappa$ : Vortex viscosity

$\rho: \quad$ Density of the fluid

$\theta$ : Dimensionless temperature

$\mu$ : Viscosity of the fluid

$\sigma$ : Microrotational component

$f$ : Velocity profile.

\section{Data Availability}

No data were used to support this study.

\section{Conflicts of Interest}

The authors declare that there are no conflicts of interest.

\section{Acknowledgments}

This work was supported by the Competitive Research Scheme (CRS) Project funded by TEQIP-III (ATU) of Rajasthan Technical University, Kota (TEQIP-III/RTU (ATU)/CRS/2019-20/03).

\section{References}

[1] A. C. Eringen, "Simple microfluids," International Journal of Engineering Science, vol. 2, no. 2, pp. 205-217, 1964.

[2] A. Eringen, “Theory of micropolar fluids," Indiana University Mathematics Journal, vol. 16, no. 1, pp. 1-18, 1966.
[3] G. Lukaszewicz, Micropolar Fluids: Theory and Applications, Brikhauser, Basel, Switzerland, 1999.

[4] A. C. Eringen, Microcontinuum Field Theories II: Fluent Media, Springer, New York, NY, USA, 2001.

[5] G. Ahmadi, "Self-similar solution of imcompressible micropolar boundary layer flow over a semi-infinite plate," International Journal of Engineering Science, vol. 14, no. 7, pp. 639-646, 1976.

[6] R. Ellahi, M. Hassan, A. Zeeshan, and A. A. Khan, "The shape effects of nanoparticles suspended in HFE-7100 over wedge with entropy generation and mixed convection," Applied Nanoscience, vol. 6, no. 5, pp. 641-651, 2016.

[7] A. S. Dogonchi and D. D. Ganji, "Study of nanofluid flow and heat transfer between non-parallel stretching walls considering Brownian motion," Journal of the Taiwan Institute of Chemical Engineers, vol. 69, pp. 1-13, 2016.

[8] S. K. Jena and M. N. Mathur, "Similarity solutions for laminar free convection flow of a thermomicropolar fluid past a nonisothermal vertical flat plate," International Journal of Engineering Science, vol. 19, no. 11, pp. 1431-1439, 1981.

[9] J. V. Ramana Murthy and J. Srinivas, "Second law analysis for Poiseuille flow of immiscible micropolar fluids in a channel," International Journal of Heat and Mass Transfer, vol. 65, pp. 254-264, 2013.

[10] J. Peddieson, "An application of the micropolar fluid model to the calculation of a turbulent shear flow," International Journal of Engineering Science, vol. 10, no. 1, pp. 23-32, 1972.

[11] D. A. S. Rees and A. P. Bassom, "The Blasius boundary-layer flow of a micropolar fluid," International Journal of Engineering Science, vol. 34, no. 1, pp. 113-124, 1996.

[12] K. Bhattacharyya, S. Mukhopadhyay, G. C. Layek, and I. Pop, "Effects of thermal radiation on micropolar fluid flow and heat transfer over a porous shrinking sheet," International Journal of Heat and Mass Transfer, vol. 55, no. 11-12, pp. 2945-2952, 2012.

[13] S. Paoletti, F. Rispoli, and E. Sciubba, "Calculation exergetic loses in compact heat exchanger passages," ASME AES, vol. 10, pp. 21-29, 1989.

[14] R. S. R. Gorla, "Second law analysis of mixed convection in a laminar, non-Newtonian fluid flow through a vertical channel," ISRN Applied Mathematics, vol. 2011, Article ID 287691, 13 pages, 2011.

[15] K. Ahmad, A. Ishak, and R. Nazar, "Micropolar fluid flow and heat transfer over a nonlinearly stretching plate with viscous dissipation," Mathematical Problems in Engineering, vol. 2013, Article ID 257161, 5 pages, 2013.

[16] S. Qayyum, T. Hayat, S. A. Shehzad, and A. Alsaedi, "Effect of a chemical reaction on magnetohydrodynamic (MHD) stagnation point flow of Walters-B nanofluid with Newtonian heat and mass conditions," Nuclear Engineering and Technology, vol. 49, no. 8, pp. 1636-1644, 2017.

[17] P. Mathur and S. R. Mishra, "Free convective Poiseuille flow through porous medium between two infinite vertical plates in slip flow regime," Pramana, vol. 94, no. 1, 2020.

[18] A. Rauf, S. A. Shahzad, M. K. Siddiq, J. Raza, and M. A. Meraj, "Mixed convective thermally radiative micro nanofluid flow in a stretchable channel with porous medium and magnetic field," AIP Advances, vol. 6, no. 3, Article ID 035126, 2016.

[19] P. Mathur and S. Mishra, "Free convective magnetohydrodynamic flow over an exponentially stretching sheet with radiation," Heat Transfer-Asian Research, vol. 48, no. 7, 2019.

[20] S. R. Mishra and P. Mathur, "Williamson nanofluid flow through porous medium in the presence of melting heat transfer boundary condition: semi-analytical approach," 
Multidiscipline Modeling in Materials and Structures, vol. 17, no. 1, pp. 19-33, 2020.

[21] L. S. Sundar, K. V. Sharma, M. K. Singh, and A. C. M. Sousa, "Hybrid nanofluids preparation, thermal properties, heat transfer and friction factor - a review," Renewable and Sustainable Energy Reviews, vol. 68, pp. 185-198, 2017.

[22] A. Hussain, A. Rehman, S. Nadeem et al., "A combined convection carreau-Yasuda nanofluid model over a convective heated Surface near a stagnation point: a numerical study," Mathematical Problems in Engineering, vol. 202114 pages, 2021.

[23] Z.-W. Tong, "NC-ND license nonlinear thermal radiation and activation energy significances in slip flow of bioconvection of oldroyd-B nanofluid with cattaneo-christov theories," 2021.

[24] S. Batool, M. Y. Malik, A. S. Alqahtani, and Q. Mahmood ul Hassan, "Double diffusion in stretched flow over a stretching cylinder with activation energy and entropy generation," Case Studies in Thermal Engineering, vol. 26, Article ID 101119, 2021.

[25] M. Awais, S. E. Awan, M. A. Zahoor Raja et al., "Effects of variable transport properties on heat and mass transfer in MHD bioconvective nanofluid rheology with gyrotactic microorganisms: numerical approach," Coatings, vol. 11, no. 2, pp. 1-19, 2021.

[26] B. K. Siddiqui, S. Batool, Q. mahmood ul Hassan, and M. Y. Malik, "Repercussions of homogeneous and heterogeneous reactions of 3D flow of $\mathrm{Cu}$-water and $\mathrm{AL2O} 3$-water nanofluid and entropy generation estimation along stretching cylinder," Ain Shams Engineering Journal, 2021.

[27] M. Hemmat Esfe and S. Esfandeh, "A new generation of hybrid-nanofluid: thermal properties enriched lubricant fluids with controlled viscosity amount," SN Applied Sciences, vol. 2, p. 1154, 2020.

[28] J. C. Umavathi, A. J. Chamkha, A. Mateen, and A. Al-Mudhaf, "Unsteady two-fluid flow and heat transfer in a horizontal channel," Heat and Mass Transfer, vol. 42, no. 2, pp. 81-90, 2005 . 